\title{
Atypical Electrophysiological Findings in a Patient with Acute Motor and Sensory Axonal Neuropathy
}

\author{
Viviana Versace ${ }^{1,2 *}$, Stefania Campostrini ${ }^{1,2}$, Frediano Tezzon ${ }^{3}$, Sara Martignago ${ }^{1,2}$, \\ Markus Kofler ${ }^{4}$, Leopold Saltuari ${ }^{1,2,4}$, Luca Sebastianelli ${ }^{1,2+}$ and Raffaele Nardone ${ }^{3,5+}$ \\ ${ }^{1}$ Department of Neurorehabilitation, Hospital of Vipiteno, Vipiteno, Italy, ${ }^{2}$ Research Unit for Neurorehabilitation South Tyrol, \\ Bolzano, Italy, ${ }^{3}$ Department of Neurology, Franz Tappeiner Hospital, Merano, Italy, ${ }^{4}$ Department of Neurology, State Hospital \\ Hochzirl, Zirl, Austria, ${ }^{5}$ Department of Neurology, Christian Doppler Medical Center, Paracelsus Private Medical University of \\ Salzburg, Salzburg, Austria
}

OPEN ACCESS

Edited by:

Ghazala Hayat,

Saint Louis University,

United States

Reviewed by:

Holli A. Horak,

University of Arizona,

United States

Vladimir Galic,

New York University,

United States

${ }^{*}$ Correspondence:

Viviana Versace

viviana.versace@sabes.it

tEqually contributing senior authors.

Specialty section:

This article was submitted to

Neuromuscular Diseases,

a section of the journal

Frontiers in Neurology

Received: 11 August 2017

Accepted: 23 October 2017

Published: 08 November 2017

Citation:

Versace V, Campostrini S, Tezzon F, Martignago S, Kofler M, Saltuari L, Sebastianelli L and Nardone R (2017) Atypical Electrophysiological Findings

in a Patient with Acute Motor and Sensory Axonal Neuropathy. Front. Neurol. 8:594. doi: 10.3389/fneur.2017.00594
Guillain-Barré syndrome (GBS) is an immune-mediated polyradiculoneuropathy with acute onset and rapid clinical worsening; early diagnosis and immunomodulating therapy can ameliorate the course of disease. During the first days, however, nerve conduction studies (NCSs) are not always conclusive. Here, we describe a 73-year-old man presenting with progressive muscular weakness of the lower limbs, ascending to the upper limbs, accompanied by distal sensory disturbances. Neuroimaging of brain and spine and NCSs were unremarkable; cerebrospinal fluid analysis revealed no albuminocytologic dissociation. Based on typical clinical features, and on positivity for serum GD1b-IgM antibodies, GBS with proximal conduction failure at multiple radicular levels was postulated, and a standard regime of intravenous immunoglobulin was administered. Four weeks later, the patient presented with flaccid tetraparesis, areflexia, and reduction of position sense, tingling paresthesias, and initial respiratory distress. Repeat NCS still revealed almost normal findings, except for the disappearance of right ulnar nerve F-waves. A few days thereafter, the patient developed severe respiratory insufficiency requiring mechanical ventilation for 2 weeks. On day 50, NCS revealed for the first time markedly reduced compound muscle action potentials and sensory nerve action potentials in all tested nerves, without signs of demyelination; needle electromyography documented widespread denervation. The diagnosis of acute motor and sensory axonal neuropathy was made. After 3 months of intensive rehabilitation, the patient regained the ability to walk with little assistance and was discharged home. In conclusion, normal NCS findings up to several weeks do not exclude the diagnosis of GBS. Very proximal axonal conduction failure with late distal axonal degeneration should be taken into consideration, and electrodiagnostic follow-up examinations, even employing unusual techniques, are recommended over several weeks after disease onset.

Keywords: Guillain-Barré syndrome, acute motor and sensory axonal neuropathy, axonal conduction failure, nodo-paranodopathy, anti-ganglioside antibodies

\section{INTRODUCTION}

Guillain-Barré syndrome (GBS) is a rapid-onset polyradiculoneuropathy leading to flaccid tetraparesis and sensory disturbances. According to pathology and electrophysiological features, GBS can be classified into acute inflammatory demyelinating polyneuropathy (AIDP) and acute motor axonal 
neuropathy (AMAN) or acute motor and sensory axonal neuropathy (AMSAN). It is now well known that "axonal" conduction failure that occurs in AMAN/AMSAN is an immune-mediated disruption of the nodal-paranodal region of the axolemma with consequent failure of saltatory conduction (1-3). The conduction failure may be reversible or may progress to distal axonal degeneration (4).

Here, we report a male patient with flaccid tetraparesis and respiratory distress in whom an acute inflammatory polyneuropathy was suspected despite lack of specific abnormal findings in repeated routine electrophysiological tests for more than 3 weeks.

\section{CASE REPORT}

A 73-year-old man was admitted to the emergency department because of progressive weakness in his lower limbs ascending to the upper limbs, accompanied by tingling sensation in his feet beginning 2 days earlier. He denied fever, infections, or diarrhea during the previous weeks.

He had a history of arterial hypertension, one-vessel coronary artery disease, chronic obliterative arteriopathy of inferior limbs, chronic obstructive pulmonary disease, and chronic nicotine exposure. Vital parameters as well as routine laboratory blood tests were normal.

Neurological examination on admission revealed distally accentuated symmetrical tetraparesis, mainly affecting ankle dorsiflexion (grade 3/5 of Medical Research Council Scale), absent deep tendon reflexes in lower limbs, diminished deep tendon reflexes in upper limbs, and hypesthesia to pinprick and vibration distal to the ankles bilaterally; plantar reflexes were normal; the patient was able to walk with assistance with foot-drop on the right side.

Cerebrospinal fluid (CSF) examination was normal on the same day. Polymerase chain reaction for neurotropic viruses in CSF was negative, as well as analysis of Borrelia burgdorferi-specific antibodies in CSF and serum.

On day 2 after admission, a cerebral contrast-enhanced magnetic resonance imaging (MRI) showed non-specific bihemispheric white matter lesions. MRI of the whole spine with gadolinium failed to show any signal alteration of the spinal cord or pathologic contrast enhancement; mild signs of degenerative lumbar spondylosis were present.

On the same day, nerve conduction studies (NCSs) of upper and lower limbs were performed using standard electrodiagnostic equipment (Viking EDX System, Natus, Middleton, WI, USA). Motor NCSs were obtained from ulnar, median, tibial, and peroneal nerves bilaterally; F-waves were elicited in ulnar and tibial nerves bilaterally, using supramaximal stimuli at wrist and ankle, respectively, at $1 \mathrm{~Hz}$ stimulation rate for eight consecutive trials; sensory NCSs were obtained from left sural and left radial nerves. All motor and sensory electroneurographies were normal, including F-waves (which were normal for minimal latency, persistence, amplitude, and morphology), except for the presence of A-waves in tibial nerves bilaterally (Figure 1, right and left median and left peroneal nerves not shown).

On day 3, anti-ganglioside antibody profile was assessed by an immunodot assay, which revealed positivity for GD1b-IgM antibodies.
The patient's clinical condition worsened, and on day 4 he presented with severe distally accentuated tetraparesis. According to Brighton's diagnostic criteria the diagnosis of GBS was postulated with a grade 2 of diagnostic certainty (5).

On day 5, a standard regime of intravenous immunoglobulin (IVIg) $0.4 \mathrm{~g} / \mathrm{kg} /$ day for 5 days was initiated. Despite IVIg therapy, the patient developed respiratory muscle weakness with nocturnal hypercapnia during the third week since admission.

On day 20, repeat CSF examination showed elevated protein and albuminocytologic dissociation (133 mg/dl).

Five days later, repeat NCS revealed absent F-waves in right ulnar nerve (Figure 2), whereas F-waves in left ulnar and both tibial nerves still showed normal persistence and normal minimal latency; A-waves were still present in tibial nerves; there was no alteration of amplitude, morphology or duration of compound muscle action potentials (CMAPs) or sensory nerve action potentials (SNAPs); motor and sensory conduction velocities were all within the normal range; no conduction blocks were detected. Repetitive stimulation at $3 \mathrm{~Hz}$ of ulnar and tibial nerves to rule out neuromuscular transmission failure did not show abnormal amplitude increment or decrement. Needle electromyography (EMG) revealed no spontaneous activity in tibialis anterior bilaterally and left dorsal interosseous; a rapid rate single-unit pattern of few motoneurons discharging at $25-30 \mathrm{~Hz}$ was detected during maximal volitional effort.

Four weeks after admission, acute respiratory insufficiency required endotracheal intubation and mechanical ventilation, followed by tracheostomy 15 days later.

The patient's condition remained stable for the ensuing 2 weeks, so that progressive weaning from ventilation was successfully possible in the seventh week. The patient was then able to breathe spontaneously through the tracheostomy without oxygen supplementation. Repeat neurological examination revealed distally accentuated flaccid tetraparesis, widespread muscle atrophy, absence of deep tendon reflexes, severe reduction of position sense distally and tingling paresthesias in all four limbs, and moderate upper limb ataxia. On day 40 after admission, the patient was successfully decanulated.

Repeat gadolinium-enhanced spinal MRI on the same day did again not show any spinal cord signal changes on T1- and T2-weighted images.

A repeat electrodiagnostic examination on day 50 revealed only slightly reduced conduction velocities, but then for the first time marked amplitude reduction without temporal dispersion of both CMAPs and SNAPs in all tested nerves; tibial nerve F-waves were still present bilaterally, with normal persistence, morphology and minimal latency; right ulnar nerve F-waves were still absent, and some A-waves were detected, left ulnar F-waves showed reduced amplitude (Figure 3). Needle EMG documented widespread spontaneous activity, subacute neurogenic restructuring of motor unit action potentials, and a reduced interference pattern.

Within 4 months of intensive neurorehabilitation, the patient regained the ability to walk with a walker and to perform activities of daily living with little assistance. He was discharged home 6 months after disease onset. 


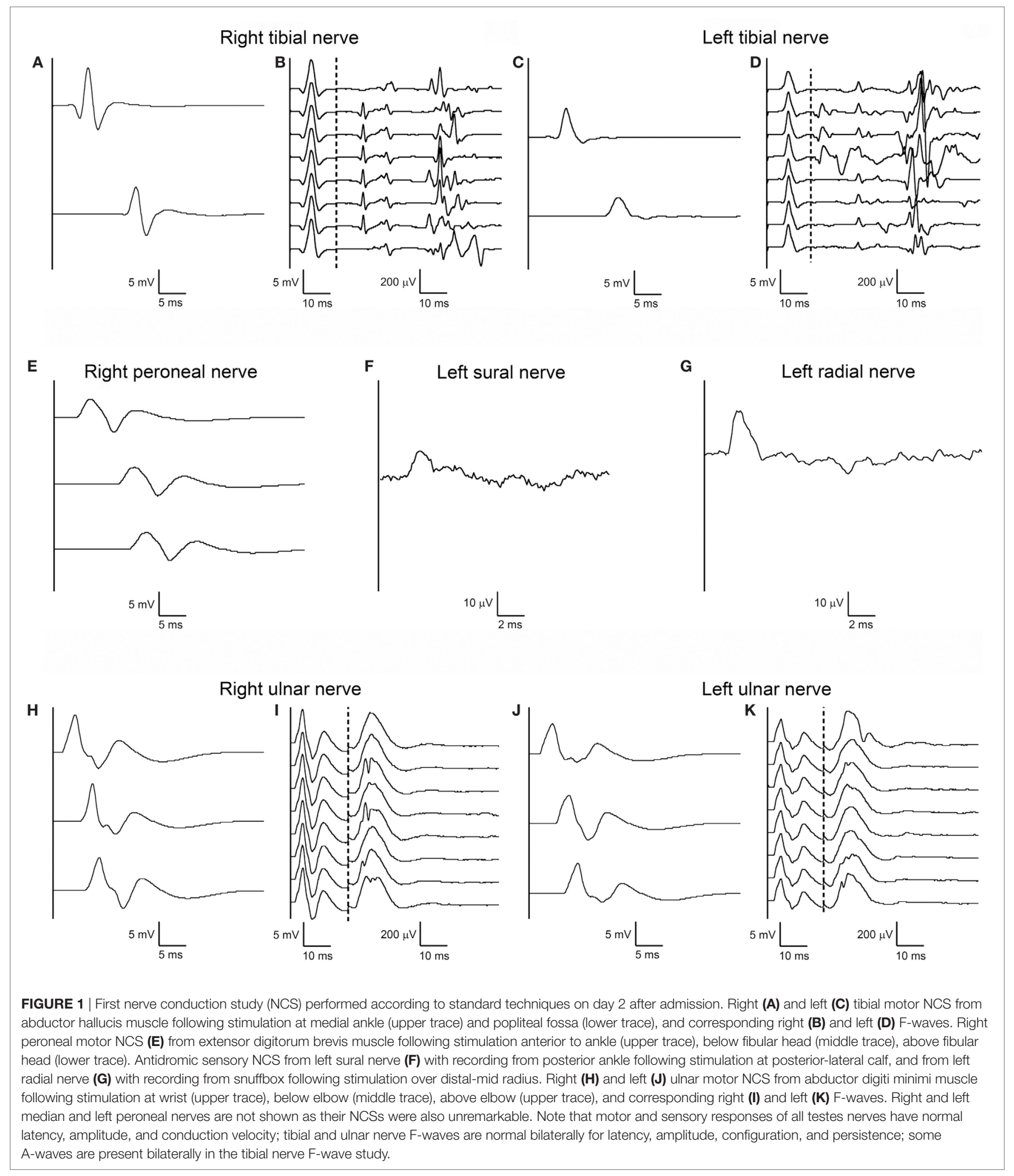

\section{DISCUSSION}

We report a patient with an AMSAN variant of GBS in whom initial diagnosis was made solely on the basis of typical clinical features and course and positivity of anti-ganglioside antibodies (GD1b-IgM) in serum, after ruling out extensive longitudinal myelitis on repeat MRI. Initial motor and sensory NCSs on days 2 and 25 after hospitalization were unremarkable, and first 


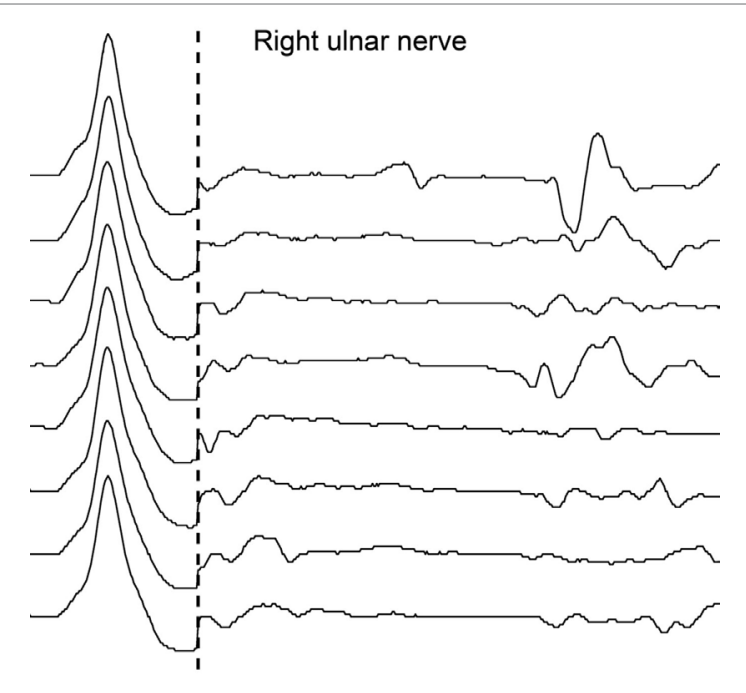

FIGURE 2 | Second nerve conduction study according to standard techniques performed on day 25 after admission. No F-waves are recorded by supramaximal stimulation of right ulnar nerve at wrist; $M$ waves are within normal range.

electrodiagnostic findings concurring with AMSAN occurred later than 4 weeks after disease onset.

The unique early abnormal finding was the presence of A-waves in recordings of late responses in tibial nerves bilaterally (Figure 1) and the absence of F-waves in the right ulnar nerve, but only in the second examination (Figure 2).

However, detection of A-waves in both tibial nerves is in fact a non-specific finding, being frequently observed in the lower extremities in elderly healthy subjects (6). The disappearance of right ulnar nerve F-waves as an isolated abnormal finding in the second NCS on day 25 was in stark contrast to the patient's serious clinical condition, and as such not sufficient to solve the diagnostic problem.

Severe flaccid ("peripheral-type") paresis in the presence of almost normal NCSs in the first two examinations up to 4 weeks from disease onset is certainly exceptional and concurs with proximal conduction failure at multiple segmental levels. At that time, there was also no evidence of axonal degeneration, based on normal CMAP and SNAP amplitudes. A recent study in a cohort of GBS patients reported normal NCS in 37\% within the first 4 days after symptom onset (7). Yet, normal NCS including normal F-waves in multiple nerves some 4 weeks after disease onset in a patient presenting with flaccid tetraparesis and respiratory distress remains difficult to explain. We assume that a substantial number of motor axons in the anterior roots must have been afflicted by conduction failure due to the immune attack. Perhaps few unblocked axons may have been sufficient to generate normal late responses. In fact, only about $5-10 \%$ of alpha-motoneurons generate $\mathrm{F}$-waves in response to an electrical stimulus (8).

It usually takes $2-3$ weeks following a proximal axonal nerve lesion for the ongoing Wallerian degeneration to reach distal sites, and to manifest as pathological spontaneous activity on EMG (9). Absence of electrophysiological signs of distal axonal degeneration some 4 weeks after disease onset remains unusual. A possible explanation could be a variable time of onset of distal axonal degeneration occurring in an immune-mediated proximal conduction failure at the nodes of Ranvier as opposed to the time course after nerve transection, particularly if the immune attack is directed only against very proximal sites $(10,11)$. Alternatively, it could be explained by the occurrence, in some proportion, of early reversible conduction failure (RCF) in the nodal region of nerve roots. The overall incidence of RCF in serially tested nerves of patients with axonal GBS variants has recently been estimated $42.8 \%$ (12). Several human and experimental studies suggest that the proportion between RCF with rapid restoration of the nodal-paranodal region, and persistent conduction failure with secondary distal axonal degeneration determine the amount of clinical recovery (4). The very favorable outcome of the herewith reported patient could be at least partly due to proximal RCF, together with efficient distal reinnervation through compensatory collateral sprouting as documented by enlarged and polyphasic MUAPs in the last EMG examination.

In two histopathological studies, very mild findings (in terms of axonal loss or fiber degeneration) were reported in some AMAN patients despite severe paralysis $(13,14)$.

Kuwabara et al. reported a retrospective series of 12 patients with GBS and reduced F-waves (persistence $<20 \%$ or total absence) as an isolated electrophysiological abnormality in two or more nerves. In their follow-up study, half of patients presented with recovery of F-waves within 2 weeks, without developing distal axonal degeneration (CMAPs remained within normal range in all consecutive examinations up to 3 months); these patients also recovered from their clinical deficits within few months. In the other half of patients, F-waves remained absent, distal CMAP amplitudes declined about 1 week after the first electrodiagnostic examination, probably because of additional lesions in distal motor nerve segments, and their clinical recovery was delayed and incomplete (15).

In the present patient, only in the third electrophysiological examination, which was performed more than 7 weeks after disease onset, and following a 3-week stay in the intensive care unit, NCS revealed significant amplitude reduction of CMAPs and SNAPs in all four limbs, without evidence of demyelination (Figure 3). Needle EMG demonstrated active axonal damage. A possible role of critical illness polyneuropathy in triggering or accelerating axonal degeneration needs to be taken into consideration, even if the occurrence of critical illness polyneuropathy in a patient with a history of mechanical ventilation, but no signs of systemic inflammatory response syndrome, sepsis, or multiorgan failure are unlikely (16).

As NCSs were not diagnostic for a long time in the course of this patient's disease, additional laboratory parameters had to be taken into consideration to establish the diagnosis. Antiganglioside antibodies GD1b of the IgM and IgG class have previously been described in association with AIDP (17), in GBS/Miller Fischer overlap syndrome patients (18) as well as in acute and chronic idiopathic sensory ataxic neuropathy (ASAN) $(19,20)$. Thus, based on the presence of these antibodies in the patient's serum, the diagnosis of AMSAN was considered, despite negative electrodiagnostic features at that time. 


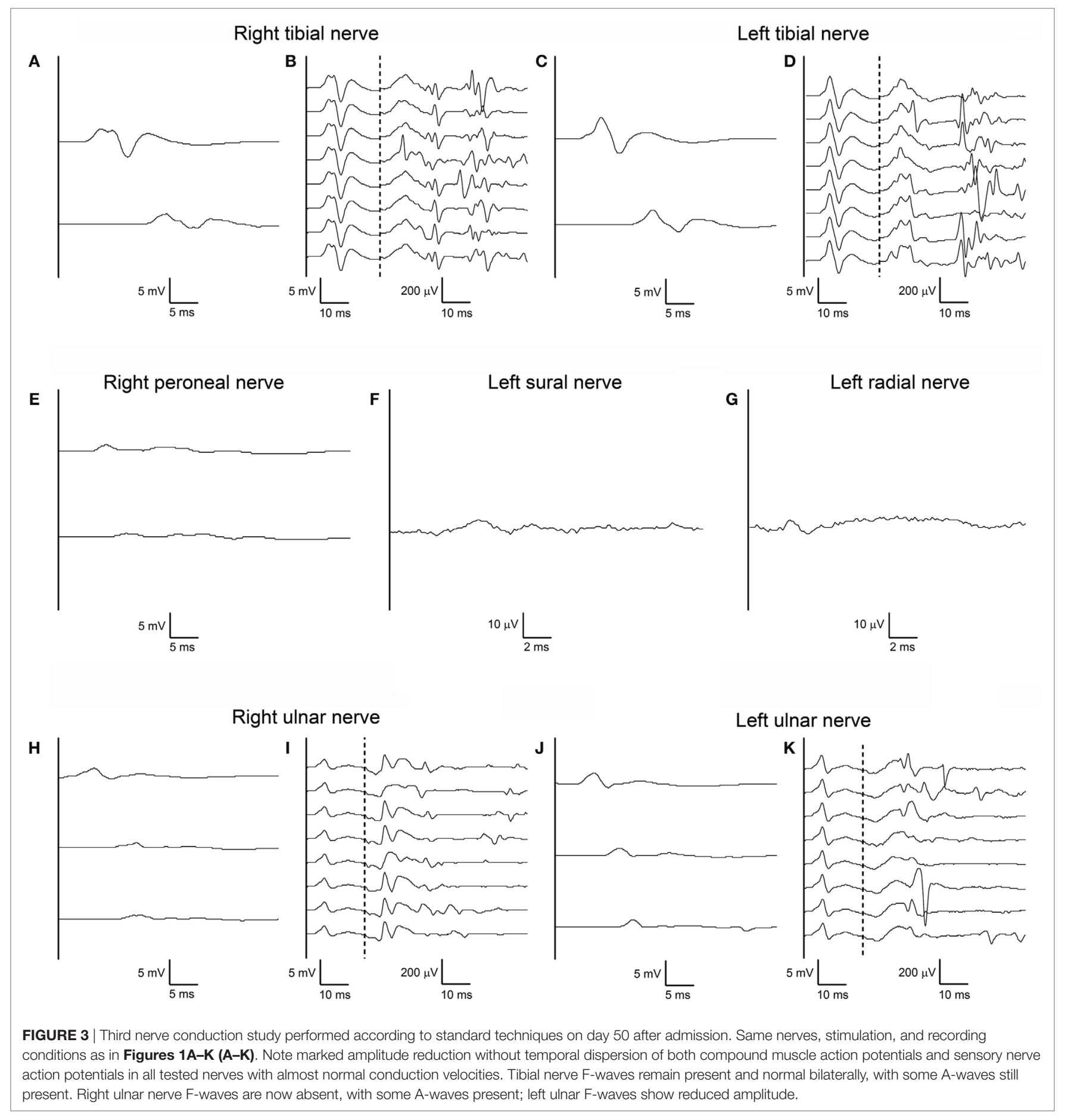

The reversible or persistent immune-mediated disruption of the nodes of Ranvier ("nodo-paranodopathy") through antibodies against gangliosides GM1 or GD1a (in AMAN and AMSAN forms) or GD1b (in ASAN forms) is recognized as underlying pathological mechanism in acute axonal polyneuropathies and determines the muscle weakness (21); the immune attack may occur along the entire peripheral nerve, but the axolemma of the nerve roots is more susceptible because of the greater permeability of the local blood-nerve barrier (22).
The occurrence of extensive Wallerian-like degeneration of large myelinated motor and sensory fibers, as well as pathological nodal lengthening, in the ventral and dorsal spinal roots of AMSAN patients was reported in pathological studies (23-25) being the exclusive pathological marker in two of these patients, who died very early (7 days) after disease onset (25).

An immune-mediated nodal disruption was also documented in dorsal roots of Gdlb immunized ASAN rabbit models (21). 


\section{CONCLUSION}

Limitations of this study-as becoming evident on retrospective-are threefold. (a) If the intervals of electrodiagnostic reexaminations would have been reduced, the first conclusive signs of axonal degeneration, i.e., amplitude reduction of CMAPs and SNAPs, as well as spontaneous activity on needle EMG, might perhaps have been detected earlier, between day 25 and day 50 after admission. (b) Attempting to assess conduction in more proximal nerve segments, i.e., proper electrical stimulation at Erb's point to activate the arm plexus may have allowed for earlier detection of decreased CMAP amplitudes due to proximal-todistal progression of axonal degeneration in upper limb nerves; furthermore, electrical cervical root stimulation, as well as the triple stimulation technique (26), may have revealed very proximal nodal-paranodal conduction failure early on. (c) Superimposed critical illness polyneuropathy_albeit unlikely_cannot entirely be ruled out in this patient.

At any rate, in selected cases with clinically suspected inflammatory neuropathy, but initially negative electrodiagnostic findings, every attempt should be undertaken, even employing

\section{REFERENCES}

1. Capasso M, Caporale CM, Pomilio F, Gandolfi P, Lugaresi A, Uncini A. Acute motor conduction block neuropathy. Another Guillain-Barré syndrome variant. Neurology (2003) 61(5):617-22. doi:10.1212/WNL. 61.5.617

2. Yuki N, Kuwabara S. Axonal Guillain-Barré syndrome: carbohydrate mimicry and pathophysiology. J Peripher Nerv Syst (2007) 12(4):238-49. doi:10.1111/j.1529-8027.2007.00153.x

3. Kokubun N, Nishibayashi M, Uncini A, Odaka M, Hirata K, Yuki N. Conduction block in acute motor axonal neuropathy. Brain (2010) 133(10):2897-908. doi:10.1093/brain/awq260

4. Uncini A, Susuki K, Yuki N. Nodo-paranodopathy: beyond the demyelinating and axonal classification in anti-ganglioside antibody-mediated neuropathies. Clin Neurophysiol (2013) 124(10):1928-34. doi:10.1016/j.clinph. 2013.03.025

5. Fokke C, van den Berg B, Drenthen J, Walgaard C, van Doorn PA, Jacobs BC. Diagnosis of Guillain-Barré syndrome and validation of Brighton criteria. Brain (2014) 137(1):33-43. doi:10.1093/brain/awt285

6. Puksa L, Stålberg E, Falck B. Occurrence of A-waves in F-wave studies of healthy nerves. Muscle Nerve (2003) 28(5):626-9. doi:10.1002/mus.10448

7. Luigetti M, Servidei S, Modoni A, Rossini PM, Sabatelli M, Lo Monaco M. Admission neurophysiological abnormalities in Guillain-Barré syndrome: a single-center experience. Clin Neurol Neurosurg (2015) 135:6-10. doi:10.1016/j.clineuro.2015.05.001

8. Bischoff C. Cap. 1.4, F-Wellen. In: Bischoff C, Dengler R, Hopf HC, editors. EMG-NLG Elektromyographie, Nervenleitungsuntersuchungen. StuttgartDeutschland: Georg Thieme Verlag KG (2014). 38 p.

9. Kimura J, Part II. Cap. 4.5 Classification of nerve injuries. In: Kimura J, editor. Electrodiagnosis in Diseases of Nerve and Muscle. Principles and Practice. USA: Oxford University Press (2013). 61 p.

10. Chaudhry V, Cornblath DR. Wallerian degeneration in human nerves: serial electrophysiological studies. Muscle Nerve (1992) 15(6):687-93. doi:10.1002/ mus.880150610

11. Löscher WN, Wanschitz J, Iglseder S, Vass A, Grinzinger S, Pöschl P, et al. Iatrogenic lesions of peripheral nerves. Acta Neurol Scand (2015) 132(5): 291-303. doi:10.1111/ane.12407

12. Uncini A, Ippoliti L, Shahrizaila N, Sekiguchi Y, Kuwabara S. Optimizing the electrodiagnostic accuracy in Guillain-Barré syndrome subtypes: unusual techniques, repeated at short intervals, and for times exceeding the commonly purported $2-3$ weeks, to arrive at a conclusive diagnosis.

\section{ETHICS STATEMENT}

Ethical approval or patient consent is not required according to national guidelines. Informed consent: a written informed consent was obtained from the patient for the publication of this case report.

\section{AUTHOR CONTRIBUTIONS}

VV performed the acquisition of data, the drafting/revising of the manuscript and accepted responsibility for conduct of research and final approval. SC, FT, and SM performed the acquisition of data and accepted responsibility for conduct of research and final approval. LuS, LeS, MK, and RN performed the drafting/revising of the manuscript and accepted responsibility for conduct of research and final approval. criteria sets and sparse linear discriminant analysis. Clin Neurophysiol (2017) 128(7):1176-83. doi:10.1016/j.clinph.2017.03.048

13. Griffin JW, Li CY, Ho TW, Xue P, Macko C, Gao CY, et al. Guillain-Barré syndrome in northern China. The spectrum of neuropathological changes in clinically defined cases. Brain (1995) 118(3):577-95. doi:10.1093/brain/118.3.577

14. Hafer-Macko C, Hsieh ST, Li CY, Ho TW, Sheikh K, Cornblath DR, et al. Acute motor axonal neuropathy: an antibody-mediated attack on axolemma. Ann Neurol (1996) 40(4):635-44. doi:10.1002/ana.410400414

15. Kuwabara S, Ogawara K, Mizobuchi K, Koga M, Mori M, Hattori T, et al. Isolated absence of $\mathrm{F}$ waves and proximal axonal dysfunction in Guillain-Barré syndrome with antiganglioside antibodies. J Neurol Neurosurg Psychiatry (2000) 68(2):191-5. doi:10.1136/jnnp.68.2.191

16. Hermans G, Van den Berghe G. Clinical review: intensive care unit acquired weakness. Crit Care (2015) 19:274. doi:10.1186/s13054-015-0993-7

17. Carpo M, Pedotti R, Allaria S, Lolli F, Matà S, Cavaletti G, et al. Clinical presentation and outcome of Guillain-Barré and related syndromes in relation to anti-ganglioside antibodies. J Neurol Sci (1999) 168(2):78-84. doi:10.1016/ S0022-510X(99)00173-2

18. Rajabally YA, Hassan-Smith G, Notturno F, Eames PJ, Hayton T, Capasso M, et al. Motor and sensory conduction failure in overlap of Guillain-Barré and Miller Fisher syndrome: two simultaneous cases. J Neurol Sci (2011) 303(1-2):35-8. doi:10.1016/j.jns.2011.01.019

19. Illa I, Rojas R, Gallardo E, Serrano C, Graus F. Chronic idiopathic sensory ataxic neuropathy: immunological aspects of a series of 17 patients. Rev Neurol (Paris) (2001) 157(5):517-22. doi:RNE-06-2001-157-5-0000-0000-101019-ART87

20. Pan CL, Yuki N, Koga M, Chiang MC, Hsieh ST. Acute sensory ataxic neuropathy associated with monospecific anti-GD1b IgG antibody. Neurology (2001) 57(7):1316-8. doi:10.1212/WNL.57.7.1316

21. Susuki K, Yuki N, Schafer DP, Hirata K, Zhang G, Funakoshi K, et al. Dysfunction of nodes of Ranvier: a mechanism for anti-ganglioside antibody-mediated neuropathies. Exp Neurol (2012) 233(1):534-42. doi:10.1016/j. expneurol.2011.11.039

22. Kiernan JA. Vascular permeability in the peripheral autonomic and somatic nervous systems: controversial aspects and comparisons with the blood-brain barrier. Microsc Res Tech (1996) 35(2):122-36. doi:10.1002/ (SICI) 1097-0029(19961001)35:2<122::AID-JEMT3>3.0.CO;2-S

23. Feasby TE, Gilbert JJ, Brown WF, Bolton CF, Hahn AF, Koopman WF, et al. An acute axonal form of Guillain-Barré polyneuropathy. Brain (1986) 109(6):1115-26. doi:10.1093/brain/109.6.1115 
24. Feasby TE, Hahn AF, Brown WF, Bolton CF, Gilbert JJ, Koopman WJ. Severe axonal degeneration in acute Guillain-Barré syndrome: evidence of two different mechanisms? J Neurol Sci (1993) 116(2):185-92. doi:10.1016/0022-510X(93)90324-R

25. Griffin JW, Li CY, Ho TW, Tian M, Gao CY, Xue P, et al. Pathology of the motor-sensory axonal Guillain-Barré syndrome. Ann Neurol (1996) 39(1): 17-28. doi:10.1002/ana.410390105

26. Taieb G, Grapperon AM, Duclos Y, Franques J, Labauge P, Renard D, et al. Proximal conduction block in the pharyngeal-cervical-brachial variant of Guillain-Barré syndrome. Muscle Nerve (2015) 52(6):1102-6. doi:10.1002/ mus. 24729
Conflict of Interest Statement: The authors declare that the research was conducted in the absence of any commercial or financial relationships that could be construed as a potential conflict of interest.

Copyright (C) 2017 Versace, Campostrini, Tezzon, Martignago, Kofler, Saltuari, Sebastianelli and Nardone. This is an open-access article distributed under the terms of the Creative Commons Attribution License (CC BY). The use, distribution or reproduction in other forums is permitted, provided the original author(s) or licensor are credited and that the original publication in this journal is cited, in accordance with accepted academic practice. No use, distribution or reproduction is permitted which does not comply with these terms. 